\title{
Bordetella pertussis Vaccine Adsorbed
}

National Cancer Institute

\section{Source}

National Cancer Institute. Bordetella pertussis Vaccine Adsorbed. NCI Thesaurus. Code C96399.

A vaccine containing acellular antigenic components of Bordetella pertussis adsorbed on a mineral carrier with active immunizing activity against pertussis infection. 